\title{
One-trial backward excitatory fear conditioning in rats: Acquisition, retention, extinction, and spontaneous recovery
}

\author{
DAVID SHURTLEFF and JOHN J. B. AYRES \\ University of Massachusetts, Amherst, Massachusetts 01003
}

\begin{abstract}
Water-deprived male albino rats received a single presentation of a 4-sec electric-grid-shock unconditioned stimulus followed by a 4-sec white-noise conditioned stimulus (a single backward conditioning trial.) Excitation conditioned to the noise was indexed in terms of the noise's subsequent ability to suppress ongoing licking of a water tube. The main findings were: (1) Excitation was acquired and was retained over a 30 -day retention interval; (2) although excitation was retained, it did not grow significantly stronger during the interval (there was no incubation effect); (3) excitation was extinguished by noise-alone trials; and (4) excitation showed more spontaneous recovery when extinction trials were separated by 29 days than when separated by only 1 day. Because these results are similar to those in the forward conditioning literature, they seem consistent with, but do not demand, the view that forward and backward excitatory conditioning involve similar learning processes. A current theory that embraces this view is opponent-process theory (Solomon \& Corbit, 1974). We suggest that opponent-process theory can (1) account for existing backward conditioning data, (2) explain the phenomenon of incubation that has previously been described in the literature while simultaneously explaining its absence in the present study, and (3) integrate certain nonmonotonic acquisition phenomena that have appeared in both the forward and backward conditioning literatures.
\end{abstract}

Reports that conditioned excitation can be established through backward conditioning procedures have for years met with skepticism (e.g., Kimble, 1961; Mackintosh, 1974; Osgood, 1953; Underwood, 1949). Indeed, backward conditioning procedures have often been used in classical conditioning experiments as control procedures designed to leave the conditioned stimulus (CS) neutral. Furthermore, when this practice was eventually criticized (Rescorla, 1967), the criticism was not that the CS might become excitatory but, rather, that it might acquire an opposite, inhibitory tendency. Such conditioned inhibition is clearly predicted by several modern theories of conditioning, including contingency theory (Rescorla, 1967) and discrepancy theory (Rescorla \& Wagner, 1972). More generally, conditioned inhibition is predicted by any theory that emphasizes the information that CSs provide about unconditioned stimuli (USs). Since backward CSs usually predict relatively long periods of US absence (e.g., Moscovitch $\&$ LoLordo, 1968), they are expected to acquire conditioned properties opposite to those of CSs that predict the imminent occurrence of USs. Empirical support

Supported by Biomedical Research Support Grant RR-07048-13. We wish to thank Paul E. Burkhardt, Michael A. Crowley, John W. Moore, Michael Vigorito, and Arnold D. Well for their comments on an earlier draft of the manuscript. Requests for reprints should be addressed to John J. B. Ayres, Psychology Department, Middlesex House, University of Massachusetts, Amherst, Massachusetts 01003. for this predicted conditioned inhibition has been accumulating in recent years and now appears substantial (Heth, 1976; Maier, Rapaport, \& Wheatley, 1976; Moscovitch \& LoLordo, 1968; Plotkin \& Oakley, 1975; Siegel \& Domjan, 1971, 1974). In addition, it has also been shown that backward (US-CS) pairings can serve to extinguish excitatory conditioning based on forward (CS-US) pairings (Ayres, Mahoney, Proulx, \& Benedict, 1976). Certainly, these findings are entirely consistent with the classic statements in the literature that the backward conditioning curve "is apparently the reverse of that present in forward conditioning [and that] forward and backward conditioning are fundamentally distinct processes" (Spooner \& Kellogg, 1947 , p. 334 , italics in the original).

Notwithstanding the above, there has also grown a body of evidence suggesting that backward conditioning can, after all, make the CS excitatory (Champion \& Jones, 1961; Heth, 1976; Heth \& Rescorla, 1973; Keith-Lucas \& Guttman, 1975; Mahoney \& Ayres, 1976; Matsumiya, 1960; Mowrer \& Aikin, 1954; Wagner \& Terry, 1975). It now appears that the crucial variable that determines whether the CS becomes excitatory or inhibitory is the number of backward pairings. With a few pairings, the CS appears to become excitatory, but, as the number of backward pairings increases, the CS appears to lose excitatory strength, eventually becoming inhibitory (Heth, 1976).

Despite the apparent solidity of the recent evidence 
for excitatory backward conditioning after a relatively small number of pairings, we were still concerned about the long history of skepticism over the reality of the effect. We were also disturbed that theories successful in many other areas would predict that only inhibition should arise from backward conditioning. We therefore thought it might be useful to obtain additional information about excitatory backward conditioning, particularly that resulting from only one backward pairing. The one-trial procedure is of special interest because it is the only procedure that provides no possibility for the CS on Trial $N$ to precede a US on Trial $\mathrm{N}+1$. In addition, it holds to an absolute minimum any possible complications that might arise from the learning of "autocontingencies" (Davis, Memmott, \& Hurwitz, 1975), that is, learning that USs predict other USs, learning that there are $x$ USs per session, etc. The specific question that we asked about one-trial backward excitatory conditioning was whether it could be retained over a substantial retention interval. There were several reasons for interest in this problem:

First, one question of interest (cf. Spooner \& Kellogg, 1947) is whether the excitatory effects of forward and backward pairings of CSs and USs really are similar. Do the two forms of apparently excitatory conditioning involve the same learning processes? If they do, then we might expect them to be affected similarly by certain parameters and to share certain basic phenomena. Indeed, it is beginning to appear that this is the case. For example, backward excitatory conditioning, like forward conditioning, increases as a function of US intensity (Burkhardt, 1980b). Also, like conditioning to a forward CS, excitatory conditioning to a backward CS can be blocked by the presence of a second, already excitatory, CS contiguous with reinforcement (Burkhardt, 1980a). Since one of the hallmarks of excitatory forward conditioning is its retention over a forgetting interval (e.g., Hendersen, 1978; Hoffman, Fleshler, \& Jensen, 1963), we might also expect backward excitatory conditioning to be similarly retained.

Second, retention over a forgetting interval is inconsistent with the possibility that the observed excitatory effects of backward-paired CSs reflect only some short-lived nonassociative process (cf. Kremer $\&$ Kamin, 1971). Although previous investigations of backward conditioning have included controls to reduce the plausibility of nonassociative interpretations of the observed conditioned response (CR), the retention of backward excitatory conditioning over a substantial forgetting interval would reduce that plausibility still further. Of course, one could still argue that retention following a backward pairing might reflect a long-term nonassociative process, just as behavioral changes produced by brain damage reflect such a process. It would be difficult to imagine, however, what the nonassociative basis of such retention might be. More important to the present authors, it is clear that a failure to find retention would certainly open to question previous associative interpretations of alleged backward excitatory conditioning (e.g., Mahoney \& Ayres, 1976).

Third, Heth (1976) has suggested the possibility that, in backward conditioning procedures, excitation and inhibition are conditioned simultaneously to the CS on each trial. Furthermore, Hendersen (1978) has found evidence to suggest that, unlike conditioned excitation, conditioned inhibition weakens over a retention interval (see, also, Thomas, 1979; Witcher, 1978). Heth's suggestion, taken together with Hendersen's findings, implies that an excitatory CR based on backward (US-CS) pairings should actually show an "incubation effect" like that sometimes seen in forward conditioning studies (e.g., Bindra \& Cameron, 1953; Frey \& Gavin, 1975; McMichael, 1966, 1968; Tarpy, 1966). That is to say, the strength of the CR should actually increase over a retention interval. This is because conditioned excitation should suffer less and less competition from (the postulated) conditioned inhibition as that inhibition weakens over the interval. (As we shall see later, there are other considerations, both theoretical and empirical, that militate against a finding of incubation following one-trial backward conditioning; we did not, however, consider these reasons sufficient to deter our looking for the phenomenon.)

Using rat subjects, we examined here the retention of excitation resulting from a single backward pairing of an electric-grid-shock US and an auditory CS. As in much of the previous work on one-trial backward fear conditioning (e.g., Burkhardt, 1980a, 1980b; Mahoney \& Ayres, 1976), we assessed excitation by presenting the CS to rats licking a water tube and by noting the disruption in licking that the CS produced. greater disruption was assumed to reflect greater excitation.

In Experiment 1, we used a within-subjects test, focusing mainly on simple retention: Rats were given a single backward pairing followed by an extinction test for conditioned excitation. After a long retention interval, the same rats were tested again. In Experiment 2, we used a between-groups test: One group was tested after a short retention interval; another group was tested after a long one. Here we focused not only on retention, but also on incubation, for, in Experiment 2, possible incubation effects could not be complicated by extinction effects. Discrepant incidental findings in Experiments 1 and 2 then suggested the possibility that spontaneous recovery of extinguished backward-conditioned excitation might be greater when two extinction tests were 1 month apart as opposed to 1 day apart. In Experiment 3, we assessed that possibility.

\section{EXPERIMENT 1}

\section{Method}

Subjects. Twenty-four male albino rats, 91 days old on arrival from the Holtzman Company, Madison, Wisconsin, were housed 
individually in a continuously lighted room. Purina Lab Chow was always available in their home cages.

Apparatus. One Gerbrands Model $\mathrm{C}$ conditioning chamber was housed in a ventilated $.61-\mathrm{m}$ cube of $12.7-\mathrm{mm}$ plywood lined with acoustical tile. The inside dimensions of the chamber were $23.2 \times$ $20.3 \times 19.5 \mathrm{~cm}$. The floor was composed of 18 stainless steel rods, $2 \mathrm{~mm}$ in diameter, mounted $1.3 \mathrm{~cm}$ apart center to center. The end walls were aluminum; the side walls and top were transparent Plexiglas. In one side wall was a $5.1 \times 1.3 \mathrm{~cm}$ aperture through which a drinking tube was placed. The tube was attached to a bottle of tap water and to a Grason-Stadler drinkometer (Model E4690A-1) that recorded licks. The CS, a white noise provided by a Grason-Stadler 455 noise generator, was presented through a $10-\mathrm{cm}$ speaker mounted on the chamber lid. The CS presentation raised the sound level in the chamber approximately $26 \mathrm{~dB}$ above the $62-\mathrm{dB}$ background. The US was a $4-\mathrm{sec} 1-\mathrm{mA}$ scrambled grid shock provided by a high-voltage, high-resistance shock source and scrambled through a relay sequencing scrambler (Hoffman \& Fleshler, 1962). Events in the experimental chamber were controlled and recorded by a computer in a nearby room.

Procedure. One week after arriving at the laboratory, the rats were assigned randomly to three groups, and the first experimental session was begun. Twenty-four hours before this session, the rats' home-cage water bottles were removed. Throughout the rest of the experiment, each rat was allowed access to water in its home cage for 30 min following removal from the experimental chamber.

The rats were run one at a time in a random order that remained constant throughout the experiment. For each of the first 4 days, all rats were allowed 110 licks on the drinking tube in the experimental chamber. Behavioral measures that were recorded included latency to first lick, time between Licks 1 and 90 , time between Licks 90 and 100, and time between Licks 100 and 110 . On Day 5 , the one-trial conditioning day, each rat was placed in the experimental chamber for $432 \mathrm{sec}$ with the water bottle absent. After $214 \mathrm{sec}$, each rat was given a 4-sec 1-mA shock US. The time of onset of a 4-sec white-noise CS, however, varied among the three groups. For Group $C(N=10)$, the CS began $184 \mathrm{sec}$ before US onset. This group was an explicitly unpaired control. (For a comparison of this control with others in the one-trial preparation, see Mahoney \& Ayres, 1976.) For Group B4 $(\mathbf{N}=7)$, the CS began $4 \mathrm{sec}$ after US onset. For Group B8 $(N=7)$, the CS began $8 \mathrm{sec}$ after US onset. (We used these US-CS intervals in an attempt to replicate the backward conditioning procedures of Mahoney and Ayres (1976). Mahoney and Ayres unexpectedly (but see Razran, 1956, p. 60) found somewhat stronger excitatory conditioning in Group B8 than in Group B4, although the difference was not reliable. We were curious to see if we would obtain a similar effect.) On Day 6, we tested for conditioning. Each rat was placed in the experimental chamber with the water bottle present. Each rat was allowed 100 licks. Then the CS was turned on until the rat licked 10 more times. Behavioral measures recorded included latency to first lick, time between Licks 1 and 90 , time between Licks 90 and 100, and time between Licks 100 and 110 . The last three measures were now defined as recovery time, pre-CS time, and CS time, respectively. Of these, the measure of main interest was the $\mathrm{CS}$ time. It was taken as an index of excitatory fear conditioning (conditioned suppression) conditioned to the CS.

Following the test session, the rats were returned to their home cages and were freely fed and watered for 28 days. On the 28 th day after the first test, their water bottles were removed; $24 \mathrm{~h}$ later, the rats were given a second test session for conditioned suppression to the white-noise CS. Altogether, then, 30 days intervened between the one backward conditioning trial and the second test for suppression.

\section{Results}

Statistical considerations. On the measures of primary interest, the CS times, we used Wilcoxon rank sums tests to make planned comparisons be- tween groups and Wilcoxon signed ranks tests to make planned comparisons within groups (e.g., Hollander \& Wolfe, 1973). Usually the direction of differences was clearly predicted from theoretical or empirical considerations; therefore, all tests involving CS times were one-tailed unless otherwise indicated. The remaining dependent measures, the pre$\mathrm{CS}$ times, the first lick latencies, and the recovery times were not expected to differ systematically across groups (cf. Burkhardt \& Ayres, 1978; Mahoney \& Ayres, 1976); accordingly, they were analyzed using two-tailed post hoc multiple comparisons for unequal sample sizes with $\alpha$ set equal to .05 experimentwise (Hollander \& Wolfe, 1973, pp. 124-129).

The top portion of Table 1 shows the median CS times on each test day following conditioning and dummy CS times on the last lick-training day before conditioning. The numbers to the right of the median show the interquartile range of each sample. On the last lick-training day, before experimental treatment, the dummy CS times were relatively short for all three groups; group differences were not reliable. On the first test day after experimental treatment, however, both Groups B4 and B8 had longer CS times than did Group $C[\operatorname{Ts}(7,10) \geqslant 88$, ps $<.01]$. As in the earlier study by Mahoney and Ayres (1976), the CS times were somewhat longer for Group B8 than for Group $\mathrm{B} 4$, but, as before, the effect was not reliable.

The results described so far merely replicate the earlier evidence for one-trial excitatory backward conditioning. Of more interest are the results of the second test day, which occurred 29 days after the first and 30 days after the single backward pairing. Here, again, both Groups B4 and B8 had longer CS times than did Group $C[T s(7,10) \geqslant 88$, ps $<.01]$. The difference between $\mathrm{B} 4$ and $\mathrm{B} 8$ was reversed relative to that on Test Day 1, but, again, was not reliable.

The table also suggests a small incubation effect for all three groups. That is, there was somewhat more suppression on Test Day 2 than on Test Day 1. The increase in the CS times over the retention interval was, however, not reliable for either of the backward conditioning groups $[\mathrm{T}(7) \geqslant 3, \mathrm{p}>.05$, twotailed] or for the two backward groups combined $[\mathrm{T}(14)=30, \mathrm{p}>.10$, two-tailed $] .{ }^{1}$ The increase was also not reliable for Group C $[\mathrm{T}(10)=12, \mathrm{p}>.10$, two-tailed].

Unlike the CS times, the pre-CS times (not shown) on Test Days 1 and 2 did not differ reliably among groups. Median pre-CS times for Groups C, B4, and B8 were $2.5,1.7$, and $2.0 \mathrm{sec}$, respectively, on Test Day 1 , and were $2.5,2.1$, and $3.6 \mathrm{sec}$, respectively, on Test Day 2. This means that the group differences of interest-the differences in the CS times-were not complicated by differences in the baseline lick rates. Analyses of the first-lick latencies and recovery times also failed to yield reliable group differences on either test day. 
Table 1

Median CS Times in Seconds for Each Group on Test Days and Dummy CS Times in the Last Lick-Training Session in Experiments 1, 2, and 3

\begin{tabular}{|c|c|c|c|c|c|c|c|c|}
\hline \multirow[b]{3}{*}{ Group } & \multicolumn{8}{|c|}{ Session } \\
\hline & \multicolumn{2}{|c|}{ Baseline-Lick Last Training } & \multicolumn{2}{|c|}{ Test 1} & \multicolumn{2}{|c|}{ Test 2} & \multicolumn{2}{|c|}{ Test 3} \\
\hline & Median & Range & Median & Range & Median & Range & Median & Range \\
\hline & \multicolumn{8}{|c|}{ Experiment 1} \\
\hline $\mathrm{C}$ & 2.7 & $2.1-4.2$ & 1.8 & $1.6-2.2$ & 3.6 & $2.3-4.9$ & & \\
\hline B4 & 2.3 & $1.5-4.4$ & 4.6 & $2.6-30.5$ & 37.6 & $7.2-96.5$ & & \\
\hline \multirow[t]{2}{*}{ B8 } & 1.9 & $1.5-3.3$ & 10.6 & $2.5-22.0$ & 15.9 & $4.2-59.4$ & & \\
\hline & \multicolumn{8}{|c|}{ Experiment 2} \\
\hline$C-1$ & 2.1 & $1.5-3.2$ & 1.8 & $1.6-4.7$ & 2.4 & $1.9-3.9$ & & \\
\hline$C-30$ & 2.1 & $1.5-4.1$ & 3.9 & $2.8-\quad 6.5$ & 15.6 & $2.1-60.1$ & & \\
\hline B-1 & 2.6 & $1.7-4.0$ & 61.2 & $16.5-163.0$ & 4.6 & $4.3-7.2$ & & \\
\hline \multirow[t]{2}{*}{ B-30 } & 2.2 & $1.8-4.2$ & 65.2 & $17.6-113.0$ & 5.1 & $2.9-12.5$ & & \\
\hline & \multicolumn{8}{|c|}{ Experiment 3} \\
\hline $\mathrm{C} 1-2$ & 1.7 & $1.5-3.7$ & 1.9 & $1.9-\quad 2.3$ & 1.8 & $1.5-2.1$ & 1.7 & $1.6-2.1$ \\
\hline $\mathrm{C} 1-30$ & 2.2 & $1.6-3.4$ & 2.0 & $1.7-\quad 3.8$ & 1.8 & $1.5-5.4$ & 1.8 & $1.6-2.4$ \\
\hline B $1-2$ & 1.7 & $1.6-1.8$ & 3.8 & $2.8-\quad 5.5$ & 2.4 & $1.7-3.1$ & 2.1 & $1.6-6.3$ \\
\hline B $1-30$ & 1.7 & $1.5-2.6$ & 3.9 & $2.9-29.1$ & 9.4 & $2.7-42.2$ & 1.6 & 1.54 .4 \\
\hline
\end{tabular}

Note-Range $=$ interquartile range of each sample.

\section{Discussion}

The new finding of this experiment is that excitatory conditioning produced by a single backward (US-CS) pairing is retained over a 30-day retention interval. This finding shows that the observed suppression to the CS does not reflect some short-lived nonassociative process such as temporary sensitization (cf. Kremer \& Kamin, 1971). It therefore supports the conclusion drawn earlier (Mahoney \& Ayres, 1976) that a single backward pairing produces a true associative effect.

The retention observed here is like that seen in experiments on forward conditioning (Hendersen, 1978; Hoffman et al., 1963). It is especially notable that retention occurred after only one backward pairing, since the retention of "incompletely learned" suppression has been of interest in the forward conditioning literature. For example, Gleitman and Holmes (1967) found retention after only four forward pairings. We are unaware of any conditioned suppression study of retention after only one pairing.

While the fact that excitatory backward conditioning is retained makes it appear to resemble forward conditioning in yet another respect (cf. Burkhardt, $1980 \mathrm{a}, 1980 \mathrm{~b}$ ), this does not prove that backward and forward conditioning reflect the same learning processes. Our results, however, are at least consistent with that possibility.

Based on the suggestion made by Heth (1976), together with the findings of Hendersen (1978), we might have expected an incubation effect over the retention interval, that is, stronger suppression after the retention interval than before it. There was indeed a tendency in this direction, but it was not reliable. There is good reason to question, however, whether our design provided the best possible test of incubation. Our rats were tested after a 1-day retention interval and then again after 30 days. The first test was an extinction test. Excitation should have been weakened by virtue of that test. Even if the postulated inhibition conditioned to the CS had weakened over the retention interval, the residual excitation remaining after the first test may not have been sufficient to produce stronger suppression on the second test than on the first. It is true that there are theoretical reasons to believe that conditioned inhibition could also have been weakened by the first test (Rescorla \& Wagner, 1972), but empirical results suggest otherwise (Witcher, 1978; Zimmer-Hart \& Rescorla, 1974). One way to remove the complicating effects of extinction on the long-retention measure is to test different groups of subjects at the two retention intervals. This was done in the following experiment.

\section{EXPERIMENT 2}

Experiment 2, like Experiment 1, attempted to test for retention of excitatory backward fear conditioning. Two groups were tested following a single backward pairing. For Group B-1, 1 day elapsed between the pairing and the first test for suppression. For Group B-30, 30 days elapsed. Both groups received a second test $24 \mathrm{~h}$ after the first. Corresponding to Groups B-1 and B-30, respectively, were Groups C-1 and C-30. These groups received the explicitly unpaired procedure given to Group $\mathrm{C}$ in Experiment 1. They then received their first test for suppression either 1 or 30 days later.

\section{Method}

Subjects and Apparatus. The subjects were 36 experimentally 
naive rats similar to those in Experiment 1 and were maintained as before. The apparatus was unchanged.

Procedure. The procedure was similar to that of Experiment 1. Half the rats, Groups B-1 and B-30, received lick training and backward conditioning identical to that given Group B4 in Experiment 1. Group B-1 was then tested for suppression on each of the next 2 days. Group B-30 was tested 30 and 31 days after conditioning. Tested on the same days as Groups B-1 and B-30, respectively, were Groups C-1 and C-30. These groups were treated identically to their corresponding B groups except that, on the conditioning day, they received the explicitly unpaired procedure given Group $C$ in Experiment 1.

Experiment 2 was conducted in two replications. The second replication began 1 week after the first began. Within each replication, all groups received their experimental treatment (conditioning) on the same calendar day. The 1- and 30-day retention tests, therefore, fell on different calendar days. Although nine rats were assigned randomly to each of the four groups, one rat died in Group C-30, reducing the size of this group to eight.

\section{Results}

The results are shown in the middle portion of Table 1. On the last lick-training day, before the groups were treated differently, their dummy CS times were all relatively short and did not differ reliably. On the first test day after experimental treatment, however, Group B-1 had longer CS times than $\operatorname{did}$ Group C-1 $[\mathrm{T}(9,9)=115, \mathrm{p}<.005]$, and Group B-30 had longer CS times than did Group C-30 [T $(8,9)$ $=46, p=.005]$. There was no evidence for an incubation effect; thus, Group B-30 did not have significantly longer CS times than did Group B-1 $[T(9,9)$ $=86, \mathrm{p}>.05]$. On Test Day 2 , the CS times were still significantly longer for Group B-1 than for Group $\mathrm{C}-1[\mathrm{~T}(9,9)=116, \mathrm{p}<.005]$, but the difference between $\mathrm{B}-30$ and $\mathrm{C}-30$ was no longer reliable $[\mathrm{T}(8,9)=$ $83, p>.05]$.

It should be noted that the CS times decreased reliably across tests both for Group B-1 and for Group B-30 [T(9) $\leqslant 5$, ps $<.025]$. This decrease contrasts with the unreliable increase across tests seen in Groups B4 and B8 of Experiment 1. The interpretation of this different pattern of results will be the subject of Experiment 3.

On both Test Days 1 and 2, the groups' pre-CS times (not shown) did not differ reliably. Median preCS times on Test Day 1 were 2.7, 1.5, 2.3, and $1.9 \mathrm{sec}$ for Groups B-1, B-30, C-1, and C-30, respectively. On Test Day 2, they were $2.1,2.9,1.7$, and $2.5 \mathrm{sec}$, respectively. Thus, the CS-time differences of interest were, again, not complicated by differences in the groups' baseline rates. The groups also did not differ reliably in terms of their first-lick latencies on either test day; however, they did differ reliably in terms of their recovery times on Test Day 2. The median recovery times on this day were $20.29,20.30$, 62.17 , and $51.24 \mathrm{sec}$, respectively, for Groups B-1, C-1, B-30, and C-30. Group B-30 took significantly longer to recover than did Group B-1.

\section{Discussion}

Like Experiment 1, Experiment 2 demonstrated clearly that the excitation arising from a single backward (US-CS) pairing can be retained over a 30-day interval. Also, like Experiment 1, Experiment 2 did not find a reliable incubation effect. Such an effect might have been expected from Heth's (1976) suggestion that excitation and inhibition are conditioned simultaneously to the backward CS, taken together with Hendersen's (1978) evidence that inhibition but not excitation is forgotton over a retention interval.

Although Experiments 1 and 2 agreed in their findings about the retention of one-trial excitatory backward conditioning, their results appeared to differ in two respects.

First, the median CS times in the backward conditioning groups were roughly 12 times Jonger in Experiment 2 than those for the comparably treated group (B4) in Experiment 1. Differences in the absolute level of suppression in successive replications of the same procedure with rats from different shipments are not unusual in this procedure (for discussion, see Burkhardt \& Ayres, 1978). Methodologically, these differences argue strongly against the use of post hoc controls in the present preparation.

Second, in Experiment 2, both groups that received backward conditioning had substantially shorter CS times in their second test session than in their first. In Experiment 1, this was not the case. The declining suppression across tests in Experiment 2 resembles results previously reported following onetrial conditioning in forward, simultaneous, and backward paradigms (Burkhardt \& Ayres, 1978; Mahoney \& Ayres, 1976); the more constant levels of suppression across tests in Experiment 1 do not. The procedural difference between the experiments was that, in Experiment 2 and in the previously published research, the two test sessions were separated by $24 \mathrm{~h}$. In Experiment 1 of this report, the test sessions were separated by 29 days.

This difference in the results of the experiments appears to be subject to three interpretations. One interpretation, which we shall call the "spontaneous recovery hypothesis," is that the first test produced extinction of conditioned excitation. This excitation showed more spontaneous recovery when the second test occurred 29 days later than when it occurred 1 day later. A second interpretation, which we shall call the "CS-termination hypothesis," is that, during the first test, the rats learned that their licking terminated the CS. In the second test, provided it was given the next day, they licked faster in order to terminate the CS sooner. However, if the test was not given until 29 days later, they forgot the relation between licking and CS termination and thus were as slow to lick in the second test as they had been in the first. A third 
hypothesis, which we shall call the "CS-exposure hypothesis," emphasizes the fact that the rats in Experiment 2 received in their first extinction test a nonreinforced CS exposure that was, on the average, about 12 times longer than that received by the rats in Experiment 1. Therefore, the rats in Experiment 2 showed more extinction on the second test than did the rats in Experiment 1. These three alternatives are evaluated in Experiment 3.

\section{EXPERIMENT 3}

Experiment 3 compared the loss of suppression across extinction tests when the tests were separated by $24 \mathrm{~h}$, as in Experiment 2, or by 29 days, as in Experiment 1. A fixed CS duration was used in testing to remove the programmed contingency between licking and CS termination. In addition, this fixed CS duration was the same for the rats that received their extinction tests separated by 1 day or by 29 days. With this new procedure, a finding of relatively retarded extinction with a long retention interval between extinction tests could not be explained in terms of the CS-exposure hypothesis or in terms of the CStermination hypothesis. Instead, such a result would support the spontaneous recovery hypothesis.

\section{Method}

Subjects and Apparatus. The subjects were $\mathbf{4 0}$ experimentally naive rats similar to those in Experiments 1 and 2 and were maintained as before. The apparatus was unchanged.

Procedure. The procedure was similar to that of Experiments 1 and 2. Half of the rats, Groups B1-2 and B1-30, received lick training similar to, and backward conditioning identical to, that given Group B4 in Experiment 1. The lick training here differed only in that the 100th lick produced a 2-min durnmy CS instead of a dummy CS that was terminated by the 110th lick. Both Groups B1-2 and B1-30 were tested for conditioning 1 day after the conditioning treatment; B1-2 then received its second test 2 days after the conditioning treatment ( 1 day after the first test). Group B 1-30 received its second test 30 days after conditioning ( 29 days after the first test). Twenty-four hours after their second test, both groups received a third test.

Corresponding to Groups B1-2 and B1-30 were two control groups, $\mathrm{C} 1-2$ and $\mathrm{C} 1-30$. These groups differed from the experimental groups only on the conditioning day. Here, they received the explicitly unpaired procedure given to the control groups in Experiments 1 and 2.

For all groups, the test procedure was identical: The 90th lick initiated a pre-CS period that was terminated by the 100th lick. The 100th lick initiated a $2-$ min CS. The time between the 100th and the 110th lick was recorded as before. Although the 110th lick no longer terminated the CS, we shall continue to refer to the time between the 100th and 110th lick as the "CS time." If the rat did not reach the 110th lick before the CS terminated, a CS time of $120 \mathrm{sec}$ was arbitrarily assigned.

There was one additional departure from the procedures of Experiments 1 and 2 . In those experiments, the rats had not been water deprived during the entire retention interval. Instead, they were deprived $24 \mathrm{~h}$ before their test for retention (Test 2 in Experiment 1; Test 1 in Experiment 2). If this practice were followed in Experiment 3 and if the effects of the water-deprivation procedure were cumulative over days, then Group B1-2 might be more thirsty in its crucial second test than Group B1-30. Group B1-2 would have experienced the water-deprivation cycle in the im- mediately preceding week, whereas Group B1-30 would have had no comparable exposure. To eliminate this difference, we reinstituted the water-deprivation cycle for Group B1-30 a week before the crucial second test.

Although 10 rats were initially assigned to each of the four groups, Groups B1-2 and C1-2 each lost one subject through experimenter error, prior to testing. No data from these two animals were used in statistical analyses. Similarly, Group B1-30 lost a rat in Test 2. Unlike the other two rats that were lost, this rat received at least Test 1 , so its data from both Test 1 and the last baseline lick-training session were included in analyses.

\section{Results}

The results are shown at the bottom of Table 1 . The table shows that on the last lick-training day, before the groups were treated differently, their dummy CS times were similar; differences among groups were not reliable. Following treatment, however, in Test 1 , the CS times tended to be longer for the backward-conditioned groups than for their controls, thus revealing more suppression in the backward groups. Group B1-2 was significantly more suppressed than its control, Group C1-2 $[\mathrm{T}(9,9)=122$, $\mathrm{p}<.005$ ], and Group B1-30 was significantly more suppressed than its control, Group C1-30 [T $(10,10)=$ $130, p<.05]$.

Of major interest in this study is the rate of extinction across Tests 1 and 2 as a function of the length of the retention interval between tests. Based on the spontaneous recovery hypothesis (and the results of Experiment 1), we would expect extinction to be relatively retarded in Group B1-30, which received the long retention interval between tests. Based on the same hypothesis (and the results of Experiment 2), we would expect relatively rapid extinction of suppression across tests in Group B1-2, which received the short retention interval between tests. Exactly this pattern of results was obtained: Group B1-2 suppressed significantly less in Test 2 than in Test 1 $[\mathrm{T}(9)=0, \mathrm{p}<.005]$, while Group B1-30 showed an unreliable increase in suppression across tests. Furthermore, on Test 2, Group B1-30 still suppressed more than did its control, Group C1-30 $[\mathrm{T}(9,10)=$ $118, p=.01]$, whereas the difference between Group B1-2 and its control, Group C1-2, was not reliable. Clearly, these results replicate within a single experiment the two different patterns of extinction found in Experiments 1 and 2.

Although the spontaneous recovery hypothesis predicts that extinction should be retarded in Group B1-30, it also predicts that extinction should ultimately occur, particularly in tests separated by only $24 \mathrm{~h}$. It is notable, therefore, that suppression for Group B1-30 did weaken significantly between Tests 2 and $3[T(9)=5, p<.025]$. This seems to complete the pattern of results predicted by the spontaneous recovery hypothesis.

During the three test days, the groups' pre-CS times (not shown) did not differ reliably. For Groups B1-2, B1-30, C1-2, and C1-30, respectively, the me- 
dian pre-CS times were as follows: Test $1-1.7,1.8$, 1.7 , and $1.7 \mathrm{sec}$; Test $2-2.0,2.4,2.1$, and $2.7 \mathrm{sec}$; Test 3-1.6, 1.9, 3.7, and $1.8 \mathrm{sec}$. As before, then, group differences in CS times were not complicated by differences in baseline rates. Analysis of the firstlick latencies and recovery times also revealed no reliable differences among groups on Test Day 1; however, the groups did differ in terms of the first-lick latencies on Test Days 2 and 3 and in terms of the recovery times on Test Day 2. Median first-lick latencies for Groups B1-2, C1-2, B1-30, and C1-30, respectively, were $22.22, .09,12.17$, and $65.91 \mathrm{sec}$ on Test Day 2, and .14, .05, 3.37, and $32.87 \mathrm{sec}$ on Test Day 3. Group C1-30 differed significantly from Group C1-2 on Test Day 2 and from Groups C1-2 and B1-2 on Test Day 3. On Test Day 2, the median recovery times for Groups B1-2, C1-2, B1-30, and C1-30, respectively, were $20.51,19.72,75.96$, and $43.56 \mathrm{sec}$. Group B1-30 took significantly longer to recover than did Groups B1-2 and C1-2. In general, the analyses of first-lick latencies and recovery times in these three experiments have not revealed a consistent pattern of results and do not lend themselves to interpretation (cf. Burkhardt \& Ayres, 1978, p. 229).

\section{Discussion}

The main results of this experiment, together with their interpretations, can be summarized as follows.

First, a CS from a one-trial backward conditioning procedure produced more suppression of licking than did a CS from an explicitly unpaired control procedure. As before (Mahoney \& Ayres, 1976), we interpret this result to mean that excitatory associative conditioning was produced by the backward conditioning procedure.

Second, following backward conditioning, suppression weakened across CS-alone test trials. For the first time in this preparation (cf. Burkhardt \& Ayres, 1978; Mahoney \& Ayres, 1976; Experiments 1 and 2, present research), this weakening could not plausibly be attributed to the learning of a lick-CS termination contingency. We interpret the weakening, therefore, as reflecting a true extinction process.

Third, as in Experiments 1 and 2, suppression after backward conditioning was retained over a 30 day retention interval. This shows that the suppression was not due to a short-lived nonassociative process, and thus adds support to the earlier conclusion that it reflects an associative process.

Fourth, the loss of suppression across CS-alone test trials was relatively retarded when those trials were separated by 29 days as compared with only 1 day. Since, in this experiment, there was no programmed contingency between licking and CS termination, this retarded extinction cannot readily be explained in terms of the CS-termination hypothesis. And, since the duration of nonreinforced CS expo- sure was constant across the two retention conditions, it also cannot be explained in terms of the CSexposure hypothesis. We therefore interpret the results in terms of the spontaneous recovery hypothesis: We conclude that the longer retention interval provided more opportunity for the spontaneous recovery of extinguished excitatory conditioning.

\section{GENERAL DISCUSSION}

It appears that backward excitatory conditioning, like forward excitatory conditioning, shows the phenomena of acquisition, extinction, spontaneous recovery, and long-term retention. It appears from other work (Burkhardt, 1980a, 1980b) that, like forward excitatory conditioning, backward excitatory conditioning increases as a function of US intensity and exhibits the phenomenon of blocking. Taken together, these findings and ours encourage the belief that forward and backward excitatory conditioning reflect the same learning processes.

The one piece of evidence from this study that might seem to speak against the conclusion that backward and forward conditioning reflect the same learning processes is the lack of a reliable incubation effect. Since incubation effects are often found in forward conditioning experiments (e.g., Frey \& Gavin, 1975; McMichael, 1966, 1968; Tarpy, 1966), one might well ask why they weren't found here. There would seem to be at least two possible reasons. First, there are no reports of incubation following only a single forward pairing. Second, incubation in forward conditioning paradigms is usually found within a 24-h retention interval with little increase in excitatory strength occurring thereafter (McMichael, 1966, 1968; Tarpy, 1966). Thus, as in forward conditioning, incubation might not be expected in our backward procedure after a 24 -h rest period.

The lack of an incubation effect in the present studies should, furthermore, not be taken as evidence against Heth's suggestion that excitation and inhibition can be conditioned simultaneously to a backward CS. Based on this suggestion, together with Hendersen's finding that inhibition but not excitation weakens over a retention interval, we had predicted an incubation effect. This prediction assumes that the inhibition conditioned to the backward CS must be substantial in order for its subsequent loss over a retention interval to have a detectable effect. The available evidence (e.g., Weisman \& Litner, 1969), however, suggests that inhibitory learning is relatively slow. Thus, even if excitation and inhibition were indeed simultaneously conditioned to a backward CS, as Heth suggested, we might not expect the inhibitory learning to be strong at all after only a single trial. Its subsequent loss, therefore, might not be detectable and might not result in measurable incubation. 


\section{A Theory of Backward Conditioning}

What kind of theory might best account for the present findings as well as, more generally, for the backward conditioning literature? Clearly, the theory must predict that excitatory associative conditioning should result from a single backward conditioning trial. It must also, however, explain why that excitation weakens with additional pairings, ultimately giving way to conditioned inhibition. The theory should, moreover, explain, if possible, the absence of an incubation effect in the present study and the presence of such effects in the literature on forward conditioning. And, while doing these things, the theory, as suggested in part by the present findings, should emphasize the identity of the processes involved in forward and backward conditioning. This implies that the theory should be able to integrate certain findings in the forward and backward conditioning literature.

As discussed in previous reports (e.g., Heth, 1976; Heth \& Rescorla, 1973; Mahoney \& Ayres, 1976; Razran, 1956), most current theories-including contingency theory (Rescorla, 1967), discrepancy theory (Rescorla \& Wagner, 1972), informational theories (e.g., Moscovitch \& LoLordo, 1968), and S-S expectancy theories, more generally-fail to meet the first requirement: They fail to predict that excitatory conditioning should result from one or more backward pairings. Although one or more of these theories might, with additional assumptions, be able to handle the data, there are two other theories whose current assumptions seem to permit them to meet many if not all of the requirements outlined above.

One of these theories (Wagner, 1976) emphasizes the notion of CS and/or US "rehearsal." Since this theory has been applied elsewhere to the prediction of the nonmonotonic backward conditioning function (Wagner \& Terry, 1975), it will not be redescribed here. We would like to note only that it is consistent with the present emphasis on the identity of learning processes involved in backward and forward conditioning. Thus, it states that the CS becomes excitatory in both procedures to the extent that joint CSUS rehearsal takes place, and it holds that, in both procedures, the CS loses excitatory strength (under some conditions becoming inhibitory) when the CS is rehearsed alone.

The second theory, the opponent-process theory of Solomon and Corbit (1974), has not, to our knowledge, been applied to the explanatory problems described above. We shall first briefly review the theory and then attempt to apply it to these problems.

According to opponent-process theory, an unconditioned stimulus evokes a process called an $a$ process. This process is postulated to recruit quickly, to be proportional to US intensity, to be constant over trials, and to die out rapidly after US termination.
Linked to the $a$ process is an opponent $b$ process. This process is initiated by the onset of the $a$ process. However, the $b$ process is sluggish: It recruits slowly, and it dies out slowly. It is not constant over trials; rather, with use, it increases in strength, it recruits faster, and it dies out even more slowly. With disuse, it decreases in strength. The manifest affective "state" of an organism is the result of the algebraic summation of these opposing processes. Stimuli contiguous with manifest A states gain control over those states. Stimuli contiguous with manifest B states gain control over those states. Conditioned suppression measures (and others, e.g., avoidance) presumably reflect these conditioned states. Conditioned A and B states, unlike unconditioned $b$ processes, are not postulated to weaken with disuse.

Let us turn now to the application of the theory to the data of backward conditioning. According to the theory, a stimulus that immediately follows electric shock for the first time would presumably be contiguous with an A state. This is because the opposing $b$ process is assumed to be very weak on Trial 1 and because some measurable (although short) period is required before the $a$ process dies out after US termination. The CS, therefore, will come to control the conditioned form of the A state. We say that the CS becomes "excitatory." The CS is not paired with the peak of the A state, however, because that peak probably occurs during the US itself. Therefore, the backward CS does not become as excitatory as a forward CS or as a simultaneous CS (Heth \& Rescorla, 1973; Mahoney \& Ayres, 1976). Furthermore, we should expect excitatory backward conditioning only under a limited set of conditions. The opponent-process theory postulates that the $a$ process dissipates quickly after US termination; so, as the gap between US termination and CS onset is increased, there should come a point at which excitatory conditioning declines rapidly. The present study and that of Mahoney and Ayres (1976) included a gap of $4 \mathrm{sec}$; this gap did not produce any detectable decline in conditioning. Clearly, a more systematic analysis of intervals longer than $4 \mathrm{sec}$ needs to be undertaken.

Having explained why the backward CS becomes excitatory after a single pairing, we now need to explain the well-known nonmonotonic backward conditioning curve; that is, why does the backward CS lose excitatory strength with further backward pairings and ultimately become inhibitory? According to the theory, this happens because the opposing $b$ process grows stronger with use. It begins to recruit more rapidly, to die out more slowly, and to increase in intensity. As training continues, therefore, the CS is paired with increasingly weaker $A$ states and then with increasingly stronger $B$ states. The CS now comes to control the B state instead of the A state. We say that the CS has become "inhibitory."

Opponent-process theory's "use" postulate ex- 
plains why the backward CS becomes inhibitory, as we have just seen. When combined with its converse, the "disuse" postulate, it also accounts for the phenomenon of incubation and helps us to understand why incubation was not found in the present study. According to the theory, a series of massed conditioning trials should make the $b$ process stronger and longer lasting. If a test trial were to follow these massed trials immediately, the $b$ process from the preceding trial would weaken the A state normally elicited by the test CS. If, however, a long retention interval were interposed between the last conditioning trial and the test trial, the $b$ process would weaken with disuse, thereby permitting a more complete elicitation of the A state on test. We could say that incubation had occurred. Incubation would not, however, be expected in a procedure like the one used in the present experiments. First, the $b$ process is postulated to be weak on Trial 1, so its presence or absence at the time of testing might make little difference. Second, the shortest retention interval used here was $24 \mathrm{~h}$. Presumably, the $b$ process aroused by shock is fully dissipated within that interval. Any further dissipation after that would be too slight to result in a detectable incubation effect.

Spooner and Kellogg (1947), having discovered that the backward conditioning curve was nonmonotonic, concluded that backward conditioning reflected processes fundamentally different from those involved in forward conditioning. In contrast, and consistent with the present results, opponent-process theory holds that the processes are identical. The $a$ and $b$ processes are involved in both kinds of conditioning. The backward procedure is merely an arrangement that permits the CS to gain control, first, over a relatively weak $A$ state and, later, over increasingly strong B states. Forward procedures usually arrange for the CS to gain control over stronger A states with less involvement of $b$ processes and B states. Nevertheless, in forward conditioning procedures, $b$ processes should still be important. We have just cited the incubation phenomenon as one possible example of their importance. Another (perhaps related) example is the following. If an A state were well conditioned to a forward CS, further trials could only strengthen the $b$ process. Any given CS trial, therefore, would be increasingly encroached upon by the $b$ process from the preceding trial. This process, growing stronger and longer lasting with use, could progressively weaken the manifest $\mathrm{A}$ state elicited by the $\mathrm{CS}$ as conditioning trials continued. Between sessions, however, the $b$ process would weaken with disuse, resulting in a strong A state on the first trial of the next session. The result would be an apparent extinction record during the maintained acquisition conditions of Session N, followed by "spontaneous recovery" at the start of Session $N+1$. Such nonmonotonic forward conditioning curves have recently been described in the fear conditioning literature (Ayres, Berger-Gross, Kohler, Mahoney, \& Stone, 1979). The fact that such nonmonotonic forward conditioning functions can be explained in terms of the same principles used to explain the nonmonotonic backward conditioning curve seems to add further support to the suggestion of the present results that the processes involved in backward and forward conditioning are the same.

To summarize, opponent-process theory holds that backward excitatory conditioning is a genuine associative effect, qualitatively identical to forward excitatory conditioning. It holds that backward and forward procedures differ only in terms of the strength of the A state with which the CS is paired and in terms of the extent to which they permit unconditioned $b$ processes and conditioned B states to influence the criterion response. It readily explains the excitatory conditioning obtained here as well as the nonmonotonic backward conditioning function obtained elsewhere. It also explains some nonmonotonic functions recently obtained in forward conditioning procedures, thus exemplifying its ability to integrate the forward and backward conditioning literatures. Finally, it provides. a framework within which the absence of an incubation effect in the present study can be explained, while it simultaneously accounts for incubation effects found elsewhere.

\section{REFERENCES}

Ayres, J. J. B., Berger-Gross, P., Kohler, E. A., Mahoney, W. J., \& Stone, S. Some orderly nonmonotonicities in the trial-by-trial acquisition of conditioned suppression: Inhibition with reinforcement? Animal Learning \& Behavior, 1979, 7, 174-180.

Ayres, J. J. B., Mahoney, W. J., Proulx, D. T., \& Benedict, J. O. Backward conditioning as an extinction procedure. Learning and Motivation, 1976, 7, 368-381.

Binnta, D., \& Cameron, L. Changes in experimentally produced anxiety with the passage of time: Incubation effect. Journal of Experimental Psychology, 1953, 45, 197-203.

BURKhardT, P. E. Attenuation of conditioned suppression in rats: The role of forward and backward conditioning during element and compound training. Unpublished master's thesis, University of Wisconsin at Oshkosh, 1980. (a)

Burkhard, P. E. One-trial backward fear conditioning in rats as a function of US intensity. Bulletin of the Psychonomic Society, 1980, 15, 9-11. (b)

Burkhardt, P. E., \& Ayres, J. J. B. CS and US duration effects in one-trial simultaneous fear conditioning as assessed by conditioned suppression of licking in rats. Animal Learning \& Behavior, 1978, 6, 225-230.

Champion, R. A., \& Jones, J. E. Forward, backward, and pseudoconditioning of the GSR. Journal of Experimental Psychology, 1961, 62, 58-61.

Davis, H., Memmotт, J., \& Hurwitz, H. M. B. Autocontingencies: A model for subtle behavioral control. Journal of Experimental Psychology: General, 1975, 104, 169-188.

Frey, P. W., \& Gavin, W. Overnight incubation of a partially conditioned eyeblink response in rabbits. Animal Learning \& Behavior, 1975, 3, 114-118.

Gleitman, H., \& Holmes, P. A. Retention of incompletely learned CER in rats. Psychonomic Science, 1967, 7, 19-20.

Hendersen, R. W. Forgetting of conditioned fear inhibition. Learning and Motivation, 1978, 9, 16-30. 
НЕтн, C. D. Simultaneous and backward fear conditioning as a function of number of CS-UCS pairings. Journal of Experimental Psychology: Animal Behavior Processes, 1976, 2, 117-129.

Heth, C. D., \& Rescorla, R. A. Simultaneous and backward fear conditioning in the rat. Journal of Comparative and Physiological Psychology, 1973, 82, 434-443.

Hoffman, H. S., \& Fleshler, M. A relay sequencing device for scrambling grid shock. Journal of the Experimental Analysis of Behavior, 1962, 5, 329-330.

Hoffman, H. S., Fleshler, M., \& Jensen, P. Stimulus aspects of aversive controls: The retention of conditioned suppression. Journal of the Experimental Analysis of Behavior, 1963, 6, 575-583.

Hollander, M., \& Wolfe, D. A. Nonparametric statistical methods. New York: Wiley, 1973.

Ke ITH-Lucas, T., \& GutTMan, N. Robust-single-trial delayed backward conditioning. Journal of Comparative and Physiological Psychology, 1975, 88, 468-476.

KimBle, G. A. Hilgard and Marquis' conditioning and learning (2nd ed.). New York: Appleton-Century-Crofts, 1961.

Kremer, E. F., \& Kamin, L. J. The truly random control procedure: Associative or nonassociative effects in rats. Journal of Comparative and Physiological Psychology, 1971, 74, 203-210.

Mackintosh, N. J. The psychology of animal learning. New York: Academic Press, 1974.

Mahoney, W. J., \& Ayres, J. J. B. One-trial simultaneous and backward fear conditioning as reflected in conditioned suppression of licking in rats. Animal Learning \& Behavior, 1976, 4, 357-362.

Maier, S. F., Rapaport, P., \& Wheatley, K. L. Conditioned inhibition and the UCS-CS interval. Animal Learning \& Behavior, 1976, 4, 217-220.

MAtsumiYa, Y. The effects of US intensity and CS-US pattern on conditioned emotional response. Japanese Psychological Research, 1960, 2, 35-42.

McMichael, J. S. Incubation of anxiety and instrumental behavior. Journal of Comparative and Physiological Psychology, $1966,61,208-211$.

McMichaEL, J. S. Conditioned suppression increases following training in pigeons. Psychonomic Science, 1968, 13, 267-268.

Moscovitch, A., \& LoLondo, V. M. Role of safety in the Pavlovian backward fear conditioning procedure. Journal of Comparative and Physiological Psychology, 1968, 66, 673-678.

Mowrer, O. H., \& Aiken, E. G. Contiguity vs. drive-reduction in conditioned fear: Temporal variations in conditioned and unconditioned stimulus. American Journal of Psychology, 1954, 67, 26-38.

Osgoon, C. E. Method and theory in experimental psychology. New York: Oxford University Press, 1953.

Plotkin, H. C., \& OAkley, D. A. Backward conditioning in the rabbit (Oryctolagus cuniculus). Journal of Comparative and Physiological Psychology, 1975, 88, 586-590.

Razran, G. Backward conditioning. Psychological Bulletin, 1956, 53, 55-69.

Rescorla, R. A. Pavlovian conditioning and its proper control procedures. Psychological Review, 1967, 74, 71-80.
Rescorla, R. A., \& Wagner, A. R. A theory of Pavlovian conditioning: Variations in the effectiveness of reinforcement and nonreinforcement. In A. H. Black \& W. F. Prokasy (Eds.), Classical conditioning II: Current research and theory. New York: Appleton-Century-Crofts, 1972.

Siegel, S., \& Domjan, M. Backward conditioning as an inhibitory procedure. Learning and Motivation, 1971, 2, 1-11.

Siegel, S., \& Domjan, M. The inhibitory effect of backward conditioning as a function of the number of backward pairings. Bulletin of the Psychonomic Society, 1974, 4, 122-124.

Solomon, R. L., \& Conвit, J. An opponent-process theory of motivation: 1. Temporal dynamics of affect. Psychological Review, 1974, 81, 119-145.

Spooner, A., \& Kellogg, W. N. The backward conditioning curve. American Journal of Psychology, 1947, 60, 321-334.

TARPY, R. M. Incubation of anxiety as measured by response suppression. Psychonomic Science, 1966, 4, 189-190.

Thomas, D. A. Retention of conditioned inhibition in a barpress suppression paradigm. Learning and Motivation, 1979. 10, 161-177.

UNDE Rwoon, B. J. Experimental psychology. New York: AppletonCentury-Crofts, 1949.

WAGner, A. R. Priming in STM: An information-processing mechanism for self-generated or retrieval-generated depression in performance. In T. J. Tighe \& R. N. Leaton (Eds.), Habituation: Perspectives from child development, animal behavior, and neurophysiology. Hillsdale, N.J: Erlbaum, 1976.

WAgner, A. R., \& Terry, W. S. Backward conditioning to a CS following an expected vs. a surprising UCS. Animal Learning \& Behavior, 1975, 3, 370-374.

Weisman, R. G., \& Litner, J. S. The course of Pavlovian excitation and inhibition of fear in rats. Journal of Comparative and Physiological Psychology, 1969, 69, 667-672.

Witcher, E. S. Extinction of Pavlovian conditioned inhibition (Doctoral dissertation, University of Massachusetts, Amherst). Abstracts International, 1978, 39, (5-B), 2550.

Zimmer-Hart, C. L., \& Rescorla, R. A. Extinction of Pavlovian conditioned inhibition. Journal of Comparative and Physiological Psychology, 1974, 86, 837-845.

\section{NOTE}

1. Because an incubation effect could have been predicted, there may have been justification for a one-tailed test here. If so, the increase across test days for Group B4 is reliable $[\mathrm{T}(7)=3$, $p<.05]$. However, since the first test trial was nonreinforced, there was also reason to predict a decline in the CS time across test days. Previous work in this laboratory (Burkhardt \& Ayres, 1978; Mahoney \& Ayres, 1976) has indeed found such a decline with forward, simultaneous, and backward conditioning procedures. So a one-tailed test does not seem warranted.

(Received for publication June 20, 1980; revision accepted October 23, 1980.) 